\title{
Bound state of two-nucleon systems in quenched lattice QCD
}

\section{Takeshi Yamazaki for PACS-CS Collaboration}

Kobayashi-Maskawa Institute for the Origin of Particles and the Universe, Nagoya University, Naogya, Aichi 464-8602, Japan

yamazaki@kmi.nagoya-u.ac.jp

We address the issue of bound state in the two-nucleon system in lattice QCD with the quenched approximation at the lattice spacing of $a=0.128 \mathrm{fm}$ using a heavy quark mass corresponding to $m_{\pi}=0.8 \mathrm{GeV}$. To distinguish a bound state from an attractive scattering state, we investigate the volume dependence of the energy difference between the ground state and the free two-nucleon state by changing the spatial extent of the lattice from $3.1 \mathrm{fm}$ to $12.3 \mathrm{fm}$. A finite energy difference left in the infinite spatial volume limit leads us to the conclusion that the measured ground states for not only spin triplet but also singlet channels are bounded. Furthermore the existence of the bound state is confirmed by investigating the properties of the energy for the first excited state obtained by $2 \times 2$ diagonalization method. The scattering lengths for both channels are evaluated by the finite volume formula derived by Lüscher.

The XXIX International Symposium on Lattice Field Theory - Lattice 2011

July 10-16, 2011

Squaw Valley, Lake Tahoe, California 


\section{Introduction}

The strong interaction dynamically generates a hierarchical structure: three quarks are bound to form a nucleon with an energy of $1 \mathrm{GeV}$, and nucleons are in turn bound to form nuclei with a binding energy of $10 \mathrm{MeV}$ or so per nucleon. This is a multi-scale physics that computational physics should explore, and lattice QCD is responsible for explaining the nature of nuclei based on first principles.

Recently, we have made a first attempt to directly construct the Helium-3 and Helium-4 nuclei in quenched QCD [1] at a rather heavy quark mass corresponding to $m_{\pi}=0.8 \mathrm{GeV}$, and successfully confirmed the formation of Helium nuclei as a bound state. After our finding of the Helium nuclei, an evidence of the $\mathrm{H}$ di-baryon bound state in $N_{f}=2+1$ and $N_{f}=3$ QCD were reported by NPLQCD [回, 3] and HALQCD 肺 Collaborations, respectively. The situation, however, is markedly different for deuteron, which is the simplest nucleus composed of two nucleons in the spin triplet channel, and yet evidence based on lattice QCD for bound state had never been reported before our paper [5]. It is already quite some time ago that a first analysis of the two-nucleon system was made in quenched QCD [6]. Much more recently, studies were made with a partially-quenched mixed action [7] and $N_{f}=2+1$ anisotropic Wilson action [8]. Extraction of the potential between two nucleons has been investigated in quenched QCD [9]. All these studies, however, tried to calculate the two-nucleon scattering lengths assuming, based primarily on model considerations with nuclear potentials, that the deuteron becomes unbound for the heavy quark mass, corresponding to $m_{\pi} \gtrsim 0.3 \mathrm{GeV}$, employed in their simulations.

To check the validity of this assumption, we need to investigate whether the bound state exists or not in the heavy quark mass region, where studies so far have been carried out, using the arsenal of methods solely within lattice QCD. If there is a bound state, the ground state energy never yield the scattering length if substituted into the Lüscher's finite volume formula [10]. In such a case, the scattering length should be obtained from the energy of the first excited state.

We carry out two types of calculations at a heavy quark mass corresponding to $m_{\pi}=0.8 \mathrm{GeV}$ in quenched QCD. The first one is a conventional analysis in which we investigate the volume dependence of the energy shift for the ground state. Different volume dependence is expected for scattering and bound states. In the second one we investigate the energy level of the first excited state employing the diagonalization method [11] to separate the first excited state from the ground state near the threshold of $2 m_{N}$. If we find the ground state slightly below the threshold and the first excited state slightly above it, then such a configuration of the two lowest levels is consistent with the ground state being a bound state and the first excited state a scattering state with almost zero relative momentum. This method was previously used in a scalar QED simulation to distinguish a system with or without a bound state [12].

Hereafter we call the analyses employed in the first and second calculations the single state and two state analyses, respectively. The results in this article have been reported in Ref. [5].

\section{Single state analysis}

We generate quenched configurations with the Iwasaki gauge action [14] at $\beta=2.416$ whose lattice spacing is $a=0.128 \mathrm{fm}$, corresponding to $a^{-1}=1.541 \mathrm{GeV}$, determined with $r_{0}=0.49 \mathrm{fm}$ 

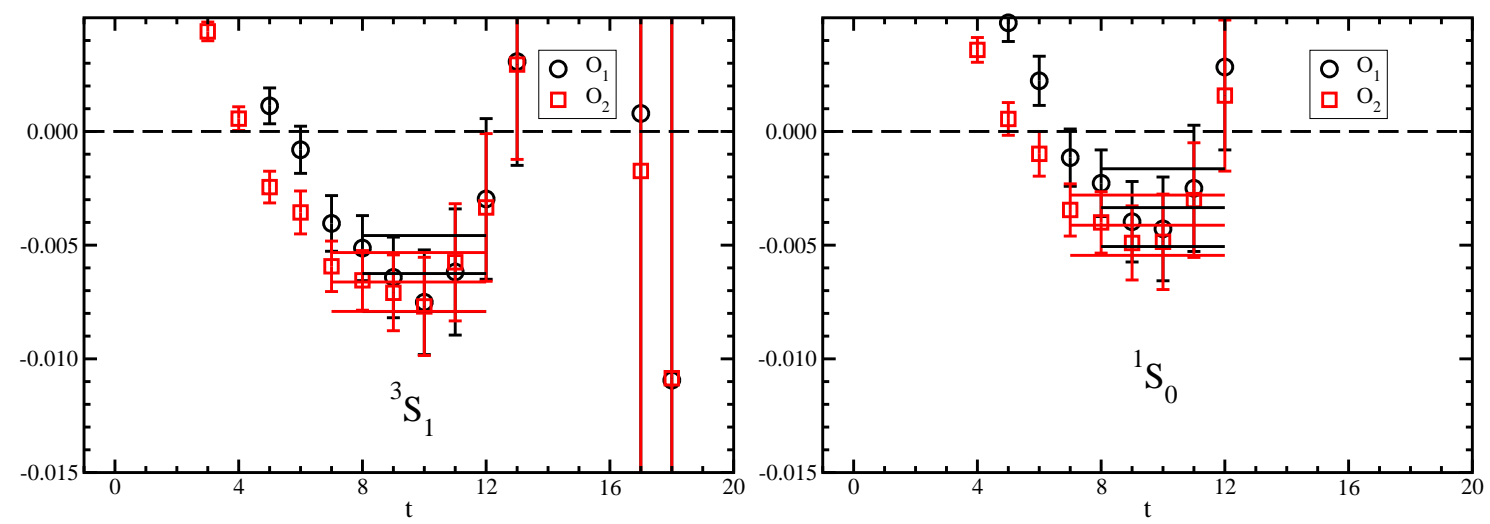

Figure 1: Effective energy shifts for ${ }^{3} \mathrm{~S}_{1}$ (left) and ${ }^{1} \mathrm{~S}_{0}$ (right) channels. Circle and square denote results for the $\mathscr{O}_{1,2}$ source operators, respectively.

as an input [15]. We take three lattice sizes, $L^{3} \times T=24^{3} \times 64,48^{3} \times 48$ and $96^{3} \times 48$, to investigate the spatial volume dependence of the energy difference between the two-nucleon ground state and twice the nucleon mass. The physical spatial extents are 3.1, 6.1 and $12.3 \mathrm{fm}$, respectively.

We use the tadpole improved Wilson action with $c_{\mathrm{SW}}=1.378$ [15]. Since it becomes harder to obtain a reasonable signal-to-noise ratio at lighter quark masses for the multi-nucleon system, we employ a heavy quark mass at $\kappa=0.13482$ which gives $m_{\pi}=0.8 \mathrm{GeV}$ for the pion mass and $m_{N}=1.6 \mathrm{GeV}$ for the nucleon mass. Statistics is increased by repeating the measurement of the correlation functions with the source points in different time slices on each configuration.

The quark propagators are solved with the periodic boundary condition in all the spatial and temporal directions using the exponentially smeared source $q^{\prime}(\vec{x}, t)=\sum_{\vec{y}} A e^{-B \mid \vec{x}-\vec{y}} q(\vec{y}, t)$ after the Coulomb gauge fixing. On each volume we employ two sets of smearing parameters: $(A, B)=$ $(0.5,0.5),(0.5,0.1)$ for $L=24$ and $(0.5,0.5),(1.0,0.4)$ for $L=48$ and 96 . The onset of ground state can be confirmed by consistency of effective masses with different sources as shown later. Hereafter the nucleon operators using the first and the second smearing parameter sets are referred to as $\mathscr{O}_{1}$ and $\mathscr{O}_{2}$, respectively.

\subsection{Numerical results}

In order to determine the energy shift $\Delta E_{L}=E_{N N}-2 M_{N}$ precisely in each volume, we define the ratio of the two-nucleon correlation function divided by the nucleon correlation function squared, $R(t)=G_{N N}(t) /\left(G_{N}(t)\right)^{2}$, where the same source operator is chosen for $G_{\mathrm{NN}}(t)$ and $G_{N}(t)$. The effective energy shift is extracted as $\Delta E_{L}^{\text {eff }}=\ln (R(t) / R(t+1))$.

In the left panel of Fig. 11 we present typical results of time dependence of $\Delta E_{L}^{\text {eff }}$ for the $\mathscr{O}_{1,2}$ sources in the ${ }^{3} \mathrm{~S}_{1}$ channel, both of which show negative values beyond the error bars in the plateau region of $t=8-11$. Note that this plateau region is reasonably consistent with that for the effective mass of the two-nucleon correlation functions. The signals of $\Delta E_{L}^{\mathrm{eff}}$ are lost beyond $t \approx 12$ because of the large fluctuations in the two-nucleon correlation functions. We determine $\Delta E_{L}$ by an exponential fit of the ratio in the plateau region, $t=8-12$ for $\mathscr{O}_{1}$ and $t=7-12$ for $\mathscr{O}_{2}$, respectively. We obtain a similar quality for the signal for the ${ }^{1} \mathrm{~S}_{0}$ channel on the $(6.1 \mathrm{fm})^{3}$ box shown in the right panel of Fig. 1. 

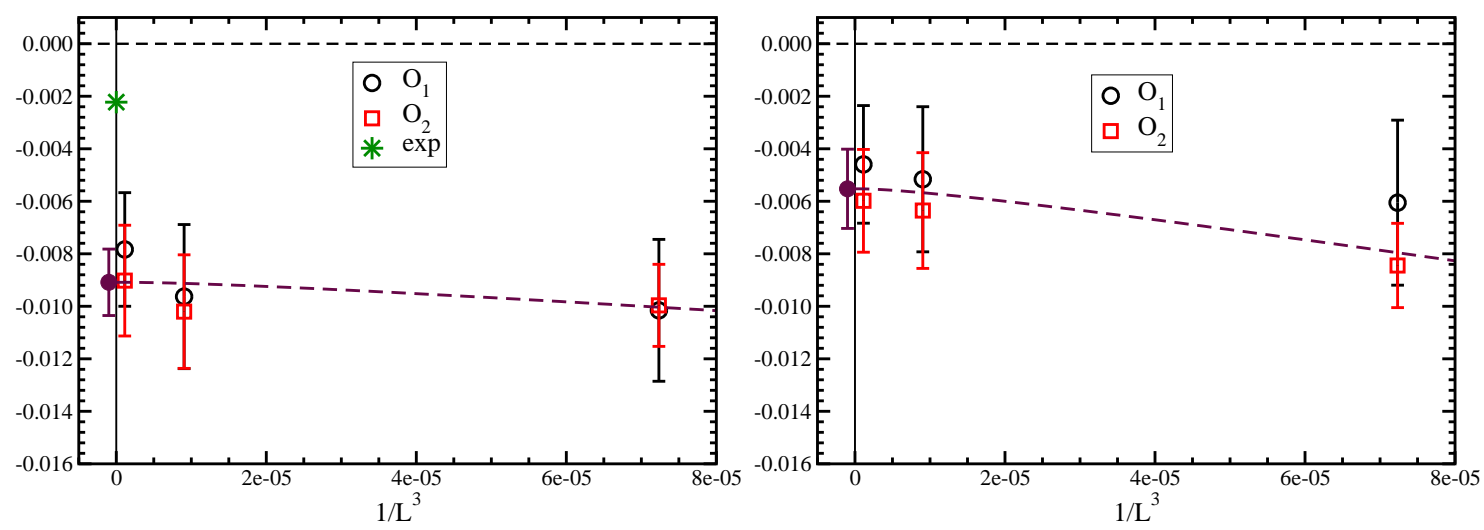

Figure 2: Volume dependence of energy shifts $\Delta E_{L}$ as function of $1 / L^{3}$ for ${ }^{3} \mathrm{~S}_{1}$ (left) and ${ }^{1} \mathrm{~S}_{0}$ (right) channels. The dashed line is extrapolation to the infinite volume, and filled circle denotes the extrapolated result.

The volume dependence of the energy shift $\Delta E_{L}$ for the ${ }^{3} S_{1}$ channel is plotted as a function of $1 / L^{3}$ in the left panel of Fig. 2. The results for the $\mathscr{O}_{1,2}$ sources are consistent within the error bars. Little volume dependence for $\Delta E_{L}$ indicates a bound state, rather than the $1 / L^{3}$ dependence expected for a scattering state, for the ground state in the ${ }^{3} \mathrm{~S}_{1}$ channel. The binding energy in the infinite spatial volume limit is extracted by a simultaneous fit of the data for the $\mathscr{O}_{1,2}$ sources employing the fit function including a finite volume effect for the two-particle bound state [12, 13],

$$
\Delta E_{L}=-\frac{\gamma^{2}}{m_{N}}\left\{1+\frac{C_{\gamma}}{\gamma L} \sum_{\vec{n}} \frac{\exp \left(-\gamma L \sqrt{\vec{n}^{2}}\right)}{\sqrt{\vec{n}^{2}}}\right\},
$$

where $\gamma$ and $C_{\gamma}$ are free parameters, $\vec{n}$ is three-dimensional integer vector, and $\sum_{\vec{n}}^{\prime}$ denotes the summation without $|\vec{n}|=0$. The binding energy, $-\Delta E_{\infty}$, is determined from $-\Delta E_{\infty}=-\gamma^{2} / m_{N}$, assuming $2 \sqrt{m_{N}^{2}-\gamma^{2}}-2 m_{N} \approx-\gamma^{2} / m_{N}$. The systematic error is estimated from the difference of the central values of the fit results choosing different fit ranges in the determination of $\Delta E_{L}$, and also using a constant fit as an alternative fit form. Adding the statistical and systematic errors by quadrature, we obtain $-\Delta E_{\infty}=9.1(1.3) \mathrm{MeV}$ for the binding energy. From the result, we conclude that the ground state in the ${ }^{3} \mathrm{~S}_{1}$ channel is a bound state.

The right panel of Fig. 2 2 plots the volume dependence of the energy shift $\Delta E_{L}$ for the ${ }^{1} \mathrm{~S}_{0}$ channel. Employing the same analysis as in the ${ }^{3} \mathrm{~S}_{1}$ channel, we find that $-\Delta E_{\infty}=5.5(1.5) \mathrm{MeV}$ in the infinite volume limit, which is $3.7 \sigma$ away from zero. This tells us that the ground state in the ${ }^{1} \mathrm{~S}_{0}$ channel is also bound at $m_{\pi}=0.8 \mathrm{MeV}$. Since the existence of the bound state in this channel is not expected at the physical quark mass, it might be a consequence of much heavier quark mass used in our calculation.

\section{Two-state analysis}

The focus of the analysis is the characteristic feature, well known from quantum mechanics, that the existence of a bound state implies a negative scattering length, and hence a scattering state just above the two-particle threshold in a finite volume. Our investigation is carried out with the diagonalization [11] of $2 \times 2$ correlation function matrix. 

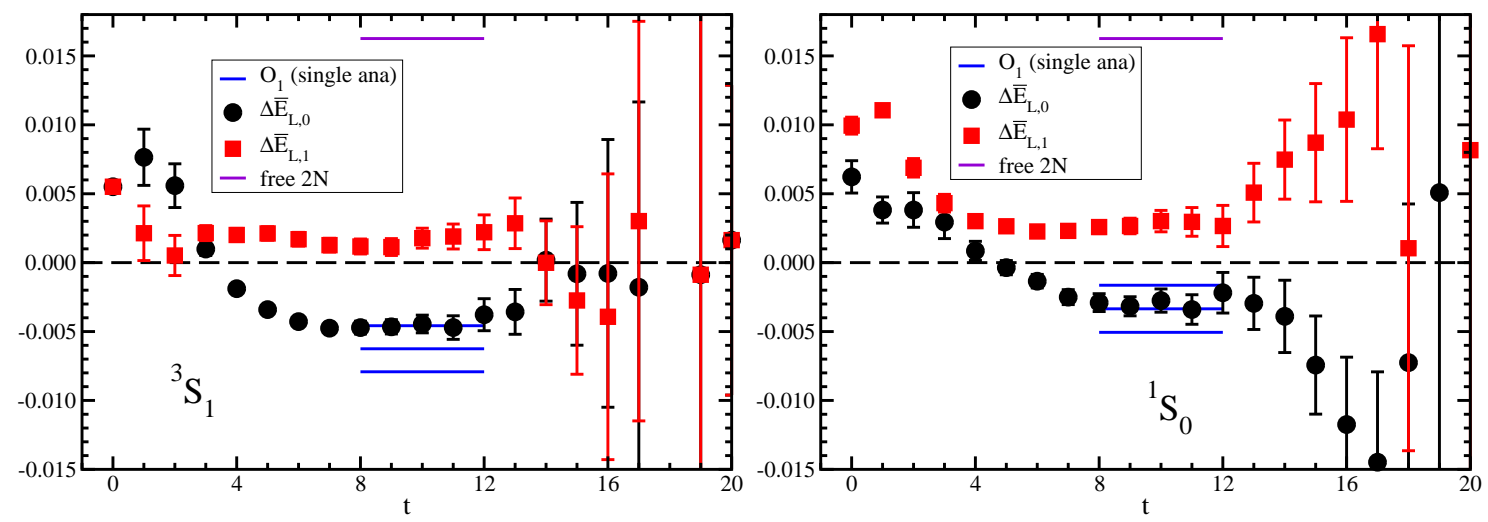

Figure 3: Effective energy shifts $\Delta \bar{E}_{L, \alpha}^{\text {eff }}$ obtained from eigenvalues for ground (circle) and first excited (square) states in ${ }^{3} \mathrm{~S}_{1}$ (left) and ${ }^{1} \mathrm{~S}_{0}$ (right) channels. Three solid lines and single line denote the result of the single state analysis with the $\mathscr{O}_{1}$ operator and free two-nucleon energy with the lowest momentum.

We work with two spatial extents, $4.1 \mathrm{fm}$ and $6.1 \mathrm{fm}$. The corresponding lattice sizes are $L^{3} \times T=32^{3} \times 48$ and $48^{3} \times 48$, respectively. The latter is the same size as in the first ensemble, but we regenerate independent configurations. Most of the simulation parameters, including the gauge and fermion actions, lattice spacing, quark mass, are identical to those explained in the previous section. The diagonalization method for the $2 \times 2$ matrix requires two operators each at source and sink time slice, which are explained in our full paper [5]. We note that due to our criteria of the choice of the operators, this analysis does not provide an independent check for the ground state energy against the single state analysis.

We diagonalize the following matrix at each $t, M\left(t, t_{0}\right)=C\left(t_{0}\right)^{-1} C(t)$, where $C(t)$ is the correlation function matrix of the two-nucleon operators and $t_{0}$ a reference time. We determine the two eigenvalues $\lambda_{\alpha}(t)(\alpha=0,1)$ of $M\left(t, t_{0}\right)$ at each $t$ and extract the energy of each eigenstate $\alpha$ through $\lambda_{\alpha}(t)=\exp \left(-\bar{E}_{L, \alpha}\left(t-t_{0}\right)\right)$. In order to determine the energy shift from the threshold as in the single state analysis, we define the ratio of the eigenvalue obtained from the diagonalization to the nucleon correlation function squared, $\bar{R}_{\alpha}(t)=\lambda_{\alpha}(t) /\left(G_{N}(t)\right)^{2}$. We also define the effective energy shift of the ratio $\bar{R}_{\alpha}$ as, $\Delta \bar{E}_{L, \alpha}^{\text {eff }}=\ln \left(\bar{R}_{\alpha}(t) / \bar{R}_{\alpha}(t+1)\right)$.

\subsection{Numerical results}

We show the results for the ${ }^{3} \mathrm{~S}_{1}$ channel on the $(6.1 \mathrm{fm})^{3}$ box. The effective energy shifts for the ${ }^{3} \mathrm{~S}_{1}$ channel are plotted in the left panel of Fig. 3. In Fig. 3 we use the nucleon correlator with the $\mathscr{O}_{1}$ operator for the denominator of $\bar{R}_{\alpha}(t)$. The ground state result $\Delta \bar{E}_{L, 0}^{\text {eff }}$ is reasonably consistent with the result of the single state analysis with the $\mathscr{O}_{1}$ source, which is expressed by the three solid lines in the figure. The first excited state is clearly higher than the ground state, but it is much lower than the free case with the lowest relative momentum, whose energy is given by $2 \sqrt{m_{N}^{2}+(2 \pi / L)^{2}}$ denoted by the single solid line in the figure.

In the right panel of Fig. 3 the effective energy shifts for the ground and first excited states $\Delta \bar{E}_{L, \alpha}^{\text {eff }}$ for the ${ }^{3} S_{1}$ channel are shown. We find features similar to those in the ${ }^{3} S_{1}$ channel. We observe that the absolute value of the energy shift of the ground state is almost half of that in the ${ }^{3} \mathrm{~S}_{1}$ channel. This is consistent with the observation in the first calculation. On the other hand, the 


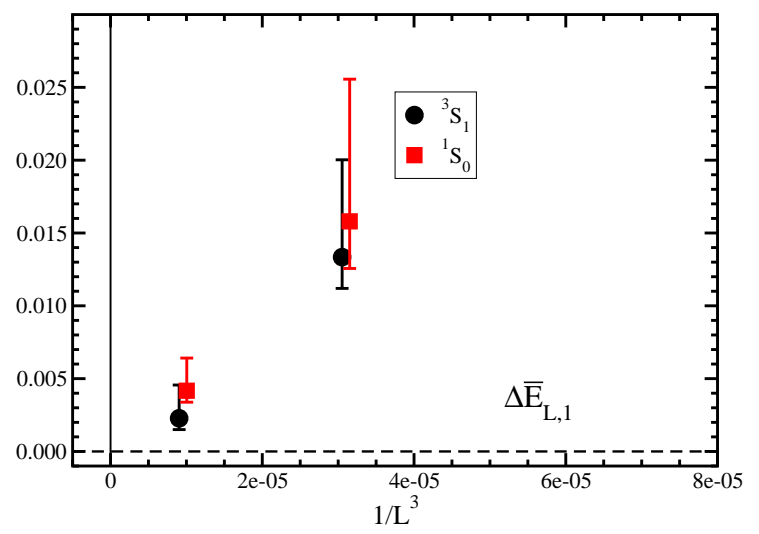

Figure 4: Volume dependence of energy shift $\Delta \bar{E}_{L, 1}$ obtained from the eigenvalues of the first excited state for ${ }^{3} \mathrm{~S}_{1}$ and ${ }^{1} \mathrm{~S}_{0}$ channels as a function of $1 / L^{3}$. The statistical and systematic errors are added in quadrature.

energy shift of the first excited state shown in the figure is almost twice larger than that in the ${ }^{3} S_{1}$ channel. This finding is consistent with the property of a system which contains a shallow bound state: The scattering length negatively increases as the binding energy decreases, diverging when the binding energy vanishes. From the results we confirm that the two-nucleon system in the both channels at the heavy quark mass of $m_{\pi}=0.8 \mathrm{GeV}$ has a bound state.

In Fig. 7 we plot the energy shift for the first excited state from the two lattice volumes as a function of $1 / L^{3}$. A roughly linear behavior, with a larger shift on the $(4.1 \mathrm{fm})^{3}$ box compared to a smaller shift on the $(6.1 \mathrm{fm})^{3}$ box, is consistent with this state being a scattering state. We evaluate the scattering length using Lüscher's finite volume formula [10], where we find reasonable consistency between the two volumes [f]]. If our finding of a bound state in quenched QCD at heavy quark mass smoothly continues to the physical point, then this is the first calculation which explained a negative scattering length for the deuteron channel.

\section{Conclusion and discussion}

We have carried out two calculations in quenched QCD to investigate whether the two nucleon systems have a bound state or not at the heavier quark mass, corresponding to $m_{\pi}=0.8 \mathrm{GeV}$. In the first calculation, we have focused on the ground state of the two-nucleon system, and have investigated the volume dependence of the energy shifts obtained with two different source operators. In the second calculation we have carried out two-state analysis using the diagonalization method with the $2 \times 2$ correlation function matrix. Based on these results we have concluded that the ground state is a bound state at the heavy quark mass in both the channels.

While similar bound state in the ${ }^{1} \mathrm{~S}_{0}$ channel was observed in recent $N_{f}=2+1$ QCD calculation at $m_{\pi}=390 \mathrm{MeV}$ [3], the existence of the bound state looks odd from the experimental point of view. We expect that the bound state vanishes at some lighter quark mass, where the scattering length diverges changing the sign from negative to positive. Further reduction of the quark mass would decrease the scattering length. Confirmation of this scenario requires to investigate the quark 
mass dependences of the binding energy and the scattering length. We leave this study to future work.

\section{Acknowledgments}

Numerical calculations for the present work have been carried out on the HA8000 cluster system at Information Technology Center of the University of Tokyo, on the PACS-CS computer under the "Interdisciplinary Computational Science Program" of Center for Computational Sciences, University of Tsukuba, and on the T2K-Tsukuba cluster system at University of Tsukuba. We thank our colleagues in the PACS-CS Collaboration for helpful discussions and providing us the code used in this work. This work is supported in part by Grants-in-Aid for Scientific Research from the Ministry of Education, Culture, Sports, Science and Technology (Nos. 18104005, 18540250, 22244018) and Grants-in-Aid of the Japanese Ministry for Scientific Research on Innovative Areas (Nos. 20105002, 21105501, 23105708).

\section{References}

[1] PACS-CS Collaboration, T. Yamazaki, Y. Kuramashi, and A. Ukawa, Phys. Rev. D81 (2010) 111504(R).

[2] NPLQCD Collaboration, S. R. Beane et. al., Phys. Rev. Lett. 106 (2011) 162001.

[3] NPLQCD Collaboration, S. R. Beane et. al., arXiv:1109.2889[hep-lat].

[4] HALQCD Collaboration, T. Inoue et. al., Phys. Rev. Lett. 106 (2011) 162002.

[5] PACS-CS Collaboration, T. Yamazaki, Y. Kuramashi, and A. Ukawa, Phys. Rev. D84 (2011) 054506.

[6] M. Fukugita, Y. Kuramashi, H. Mino, M. Okawa, and A. Ukawa, Phys. Rev. Lett. 73 (1994) 2176; M. Fukugita, Y. Kuramashi, M. Okawa, H. Mino, and A. Ukawa, Phys. Rev. D52 (1995).

[7] S. R. Beane, P. F. Bedaque, K. Orginos, and M. J. Savage, Phys. Rev. Lett. 97 (2006) 012001.

[8] NPLQCD Collaboration, S. R. Beane et. al., Phys. Rev. D81 (2010) 054505.

[9] N. Ishii, S. Aoki, and T. Hatsuda, Phys. Rev. Lett. 99 (2007) 022001; S. Aoki, T. Hatsuda, and N. Ishii, Prog. Theor. Phys. 123 (2010) 89; S. Aoki, T. Hatsuda, and N. Ishii, Comput. Sci. Dis. 1 (2008) 015009

[10] M. Lüscher, Commun. Math. Phys. 105 (1986) 153; M. Lüscher, Nucl. Phys. 3354 (1991) 531.

[11] M. Lüscher and W. Wolff, Nucl. Phys. B339 (1990) 222.

[12] S. Sasaki and T. Yamazaki, Phys. Rev. D74 (2006) 114507.

[13] S. R. Beane, P. F. Bedaque, A. Parreno, and M. J. Savage, Phys. Lett. B585 (2004) 106-114.

[14] Y. Iwasaki, Report No. UTHEP-118 (1983) (unpublished).

[15] CP-PACS Collaboration, A. Ali Khan et. al., Phys. Rev. D65 (2002) 054505. 